\title{
Planificación en la gestión de seguridad industrial e higiene ocupacional
}

\section{Planning in the management of industrial safety and occupational hygiene}

Boreidy Jiménez

boreidyjimenez@gmail.com https://orcid.org/ 0000-0003-4190-1027

Universidad del Zulia, Maracaibo - Venezuela

Palabras clave La investigación tuvo como objetivo describir el proceso de planificación en la gestión de seguridad industrial e higiene ocupacional en la industria petrolera venezolana. La metodología fue de tipo descriptiva, bajo un diseño de campo no experimental, y transeccional. La población estuvo conformada por las gerencias de Servicio Lacustre de la división occidente. Para la recolección de datos se utilizó la encuesta mediante un cuestionario, el mismo quedó conformado por 9 ítems con escala dicotómica. La validez se realizó a través del juicio de expertos, y su confiabilidad por el método de Küder - Richardson, donde se obtuvo 0,85. El análisis de los datos se realizó mediante la estadística descriptiva, a través del estudio de las frecuencias relativas. Se concluyó que estas gerencias poseen moderada presencia de las políticas, objetivos, leyes y normas inmersos en el proceso de planificación en la gestión de seguridad industrial e higiene ocupacional.

\section{Palabras clave}

Gerencia; Gestión de riesgos; Políticas; Objetivos; Leyes; Normas

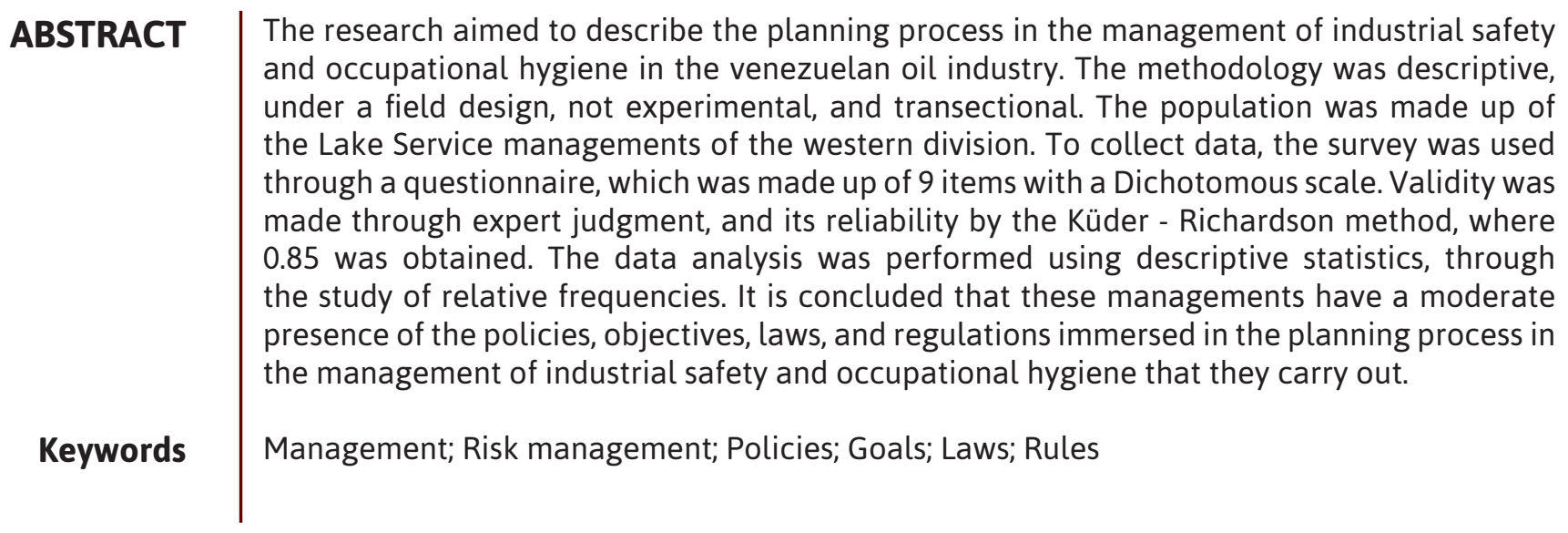




\section{INTRODUCCIÓN}

La tendencia mundial hacia la globalización, ha hecho que las diversas organizaciones adopten un proceso de gestión de seguridad industrial apegado a los lineamientos y principios de acuerdo a Normas Internacionales. De hecho, en la actualidad, las empresas del mundo conscientes de su responsabilidad social definen su política de desarrollo tomando en consideración el factor ambiental y de seguridad.

En tal sentido, se adoptan una serie de políticas de seguridad industrial, ambiente e higiene ocupacional dirigidas a garantizar la eficiencia organizacional, conforme a la cual, la seguridad de los empleados, la protección del ambiente y la higiene de los trabajadores constituyen una de las principales bases de la fuerza laboral.

Según Duque (2010), la seguridad industrial e higiene ocupacional tiene como finalidad principal la conservación de la salud de los trabajadores, lo cual requiere básicamente de un programa de protección de salud, prevención de accidentes y enfermedades profesionales, y forzosamente se extiende más allá de los límites de la mera prevención, incluyendo el aspecto más amplio de la salud total del trabajador. Es decir, se encarga del estudio de los riesgos y peligros inherentes a la actividad laboral.

Desde otra perspectiva, Burgos (2008), expresa que la seguridad industrial e higiene ocupacional es la disciplina que estudia las condiciones y organización del trabajo para reconocer, evaluar y controlar los riesgos sobre cargas existentes en los centros de trabajo, con el conocimiento de los riesgos se pueden controlar los peligros existentes con el fin de minimizarlos o eliminarlos. También se encarga de verificar las distintas variables tanto físicas, ambientales y mentales que puedan llegar a afectar la salud del trabajador de manera permanente o temporal.

Es así, como los temas tratados al respecto, llevaron al Congreso estadounidense a promulgar en 1970 una Ley de Seguridad e Higiene Laboral, mejor conocida como OSHA. La referida ley, fue diseñada para asegurar, en la medida de lo posible, que todos los hombres y mujeres estadounidenses tuvieran condiciones laborales sanas y seguras, así como para preservar los recursos humanos.

Igualmente, en Colombia, la corporación AVANTEL S.A. (2010) aplica políticas de seguridad industrial, salud ocupacional y medio ambiente, enfocadas en satisfacer las necesidades del sector corporativo y del estado colombiano. Esta organización es consciente de la necesidad de proteger la salud y seguridad de sus trabajadores en el desempeño de sus funciones, de respetar, proteger el medio ambiente del impacto derivado de las actividades de la compañía y de evitar el daño a la propiedad de terceros que se pueda generar de las mismas.

En Venezuela a partir del año 1968, se promulga el Reglamento de las Condiciones de Higiene, Seguridad en el Trabajo, y no es hasta el año 1974 cuando se pone en vigencia el decreto número 46 el cual se conoció como el Reglamento de Prevención de Incendios (Villegas, 2008). Por lo que la Comisión Venezolana de Normas Industriales (COVENIN) prosigue su intensa labor de estandarización de normas en materia de seguridad.

A pesar del desarrollo que tuvo la seguridad a nivel nacional no es sino hasta el año 2005 cuando se promulga la Ley Orgánica de Prevención, Condiciones y Medio Ambiente de Trabajo (LOPCYMAT, 2005). La misma cuenta con una filosofía de seguridad dirigida a evitar daños humanos, materiales e incidentes, así como concienciar a las personas sobre la seguridad y evitar la degradación de los recursos naturales.

En este sentido, Villegas (2008) expone que la Ley Orgánica de Prevención de Condiciones y Medio Ambiente de Trabajo (LOPCYMAT) garantiza las condiciones y medio ambiente de trabajo a los empleados y trabajadores, tanto 
de empresas públicas como privadas, previendo en su normativa sanciones pecuniarias bastante onerosas para el patrono que incumpla.

Además, la Constitución Nacional de la República Bolivariana de Venezuela (1999) en su artículo 87, expresa claramente la obligación de todo empleador o patrono de garantizar las condiciones de la seguridad, higiene y ambiente de trabajo adecuadas para el desempeño de cada trabajador. Destacando también en este apartado el hecho de la ratificación de convenios internacionales de la Organización Internacional del Trabajo (OIT), en materia de seguridad e higiene en el trabajo.

De allí pues, las empresas venezolanas se han ocupado de adoptar las políticas de seguridad exigidas por el gobierno nacional, las cuales le aseguran una gestión confiable, con la finalidad de aprovechar al máximo los recursos disponibles para prevenir accidentes y controlar los resultados obtenidos en la producción.

Enmarcada en este contexto, la industria petrolera, en específico las gerencias de servicio lacustre, ha venido administrando el control de los riesgos en materia de seguridad, higiene, salud en el trabajo y ambiente a través del sistema de gerencia integral de riesgos (SIR); siendo aprobada esta para su implantación en abril del 2004, por la junta directiva de Petróleos de Venezuela S.A. (PDVSA, página oficial de internet 2019), a fin de establecer un conjunto de requisitos interrelacionados para minimizar y controlar los riesgos relacionados a los procesos y actividades en materia de seguridad, higiene, salud en el trabajo y ambiente.

Cabe considerar que en la empresa PDVSA, según los informes revisados por la investigadora, el compromiso gerencial está dirigido principalmente a la producción, dejando de lado el bienestar físico y mental de los trabajadores (as), corriendo el riesgo de que se presenten eventos imprevistos o no deseados que interrumpen el desarrollo normal de una actividad originando las siguientes consecuencias: lesiones personales, daños materiales, afectación al ambiente y pérdidas económicas.

Es por eso por lo que la investigadora por pertenecer a una de las gerencias objeto de estudio, revisó los resultados obtenidos en las auditorías del 2019 en materia de seguridad, evidenciando debilidad en su cumplimiento. Esto se ve reflejado en los índices estadísticos de los últimos cuatro años, ya que la cantidad de eventos reportados, con lesiones al personal propio o contratado, ocurridos en todas las actividades operacionales y administrativas cada día se incrementan. Aproximadamente el $94 \%$ de los accidentes con lesiones ocurren en los sitios laborales.

Además del registro estadístico, se ve reflejado el desconocimiento de la política de seguridad, higiene y salud en el trabajo por parte de los trabajadores a pesar de la realización de campañas de divulgación mediante la entrega de un carnet donde se especifica la misma; adicionalmente se caracterizan los riesgos por áreas o proceso y se establecen las medidas de control. Sin embargo, en las inspecciones en campo se observan condiciones inseguras y los controles tardan hasta meses para ser ejecutados.

Por otra parte, es importante resaltar que PDVSA, posee procedimientos documentados para cada una de sus operaciones sin embargo en las investigaciones de los eventos, un gran porcentaje de los resultados (causas raíz) está relacionado con el desconocimiento o violación de dichos procedimientos, lo que se traduce en deficiencias en su divulgación a los trabajadores por parte del personal supervisor.

También se resalta que la empresa anualmente establece el plan operativo anual (POA) con el fin de lograr los objetivos y metas, que incluye la asignación de responsabilidades, niveles de autoridad, recursos, medios y plazos, así como las revisiones a intervalos regulares para adecuarlos 
de ser necesario; sin embargo, al finalizar cada período se detectan fallas y brechas que no logran ser completadas y que deben ser replanificadas, producto del poco compromiso del nivel gerencial y la apatía de los trabajadores quienes deben conformarse para elegir delegados y constituir los comités, pero se niegan a hacer cumplir este derecho que por ley les corresponde.

La deficiencia en la entrega de los equipos de protección personal, la falta de pericia y el desconocimiento de las responsabilidades en materia de seguridad industrial, así como de las leyes y normas que la regulan, por parte del personal supervisor acentúan la falta de cultura preventiva en cada uno de los trabajadores (as), quienes deben iniciar por comprender que la seguridad no depende de otros, sino que es un valor de vida que cada uno debe internalizar y poner en práctica.

Bajo esta óptica, vale resaltar la conveniencia de realizar un estudio que permita describir el proceso de planificación en la gestión de seguridad industrial e higiene ocupacional en la industria petrolera venezolana.

\section{Gestión de seguridad industrial e higiene ocupacional}

Es una actividad profesional tendiente para establecer los objetivos y medios de su realización, a precisar la organización de sistemas, a elaborar la estrategia del desarrollo y a ejecutar la gestión del personal, es decir, es un proceso muy particular consistente en las actividades de planeación, organización, ejecución y control, desempeñadas para determinar y alcanzar los objetivos señalados con recursos humanos y otros.

De acuerdo con Peralta (2010), la gestión de seguridad industrial e higiene ocupacional se ocupa de proteger la salud de los trabajadores, controlando el entorno del trabajo para reducir o eliminar riesgos, con respecto al ambiente toma en cuenta los aspectos físicos que rodean al trabajador mientras desempeña su cargo, en relación a la higiene ocupacional se encarga de proteger y promover la salud y el bienestar de los trabajadores, salvaguardando la comunidad así como el medio ambiente en general.

Por otra parte, Aguilar (2011) manifiesta que la gestión de seguridad industrial e higiene ocupacional es la ciencia y arte dedicados al reconocimiento, evaluación y control de aquellos factores ambientales o tensiones emanados o provocados por el lugar de trabajo y que pueden ocasionar enfermedades, destruir la salud y el bienestar o crear algún malestar significativo entre los trabajadores o los ciudadanos de una comunidad.

De igual manera, Camisón et al., (2007, p. 108), refieren que la gestión de seguridad industrial e higiene ocupacional como "una herramienta de gestión que regula y normaliza el trabajo rutinario, así como repetitivo que ponen en valor el conocimiento de las circunstancias particulares de tiempo y lugar que poseen los operarios para la ejecución segura del trabajo".

En este orden de ideas, Chiavenato (2001) considera que la higiene en el trabajo es preventiva, ya que se dirige a la salud y el bienestar del trabajador para evitar que éste se enferme o ausente de manera temporal o permanente del trabajo.

De igual manera, Chinchilla (2011, p. 25) señala la inevitable presencia de peligros en los ambientes de trabajo, afectando no solo la salud, sino también el rendimiento laboral. Basado en esto, define la seguridad industrial e higiene ocupacional como "el conjunto de técnicas y procedimientos que tienen por objeto eliminar 0 disminuir el riesgo de accidentes laborales. En este sentido, el autor explica que la seguridad industrial se ocupa de estar pendiente de los peligros que inciden en el ambiente de trabajo, tales como riesgos eléctricos, caída de objetos pesados, deficientes condiciones de orden y limpieza en 
las áreas laborales, incendios, inundaciones, entre otros riesgos, dependiendo de la actividad a ser desarrollada en la empresa.

Según la norma venezolana COVENIN 2260 (2004, p. 2), la seguridad industrial es "un conjunto de principios, leyes, criterios y normas formuladas, cuyo objeto fundamental es controlar el riesgo de accidentes que pudiesen derivar en lesiones a las personas, al igual que daños a los materiales y equipos intervinientes en el desarrollo de toda actividad productiva".

Mientras que en esta misma norma COVENIN 2260, la higiene ocupacional se define como:

La ciencia dedicada al conocimiento, evaluación y control de aquellos factores ambientales o tensiones emanadas o provocadas por o con motivo del trabajo pudiendo ser causantes de enfermedades, afectar la salud y el bienestar, o crear algún malestar significativo entre los trabajadores o los ciudadanos de la comunidad (COVENIN 2260, 2004, p. 1).

Para Hernández et al. (2005), la higiene ocupacional es una herramienta importante dentro de las organizaciones ya que facilita la prevención de accidentes y enfermedades profesionales, coordinando para cada área de trabajo, las medidas preventivas dirigidas a minimizar los riesgos de accidentes dentro del área laboral. Es importante resaltar que la seguridad industrial e higiene ocupacional, permite a la organización brindar a los trabajadores cursos de adiestramiento en el uso de los materiales para evitar accidentes, charlas de prevención, guías o folletos donde se detallen los pasos para realizar las tareas programadas y controlar el cumplimiento de las normativas en seguridad.

Los peligros que actualmente se presentan en la industria son los llamados factores de riesgos, que pueden causar daños a la salud del personal, así como también malestar e ineficiencia significativos en los trabajadores (Hernández et al., 2005), es por ello la importancia de una buena gestión de seguridad industrial e higiene ocupacional que capacite e informe a los trabajadores.

Adicionalmente, Rodríguez (2010) profundiza sobre el tema de la seguridad e higiene industrial, señalando la necesidad en las organizaciones de la correcta ejecución de las actividades, dentro de un ambiente seguro, minimizando los riesgos que afecten la salud física y mental de sus trabajadores, capacitando al personal, definiendo mejor las actividades a desarrollar de acuerdo a sus capacidades, lo cual es posible, a través del establecimiento de normas que deben desarrollarse considerando la presencia de los riesgos laborales, por tanto, el autor explica que el análisis de riesgos y accidentes constituye una actividad ligada a toda función administrativa, pues está en juego la salud del recurso humano.

Esta actividad debe ser de especial atención desde el momento en el cual se selecciona al personal, pues sus capacidades determinarán los límites de su desarrollo en la empresa, sin poner en riesgo su salud y seguridad. Aunado a esto, un personal bien capacitado, remunerado adecuadamente, tendrá mayores motivos para realizar sus actividades con mayor cuidado y entusiasmo.

Al respecto, Moreno (2010) explica que la seguridad industrial e higiene ocupacional, consiste en:

Prevenir y limitar los riesgos en el trabajo, así como establecer las normativas orientadas a la protección contra accidentes y siniestros capaces de producir daños o perjuicios al trabajador, debiendo participar en estas acciones, tanto la empresa como los trabajadores para garantizar el desarrollo de las labores en un ambiente seguro y saludable (Moreno, 2010, p. 27). 
De acuerdo con Hernández (2005), el hombre a lo largo de la historia, se ha visto acompañado del accidente. Al ejecutar las actividades productivas es evidente que el riesgo atenta contra su salud y bienestar. Conforme se ha ido haciendo más compleja la realización de las actividades de producción, se han multiplicado los riesgos para el trabajador, siendo causantes de numerosos accidentes y enfermedades afectando su estado de salud e interrumpiendo su productividad, lo cual acarrea pérdidas económicas, tanto para la empresa como para los trabajadores afectados.

Por tal motivo, se plantea que la dirección empresarial se ha interesado cada vez más en el control de las causas de los accidentes laborales, así como en reducir los riesgos de las actividades a los que se exponen sus trabajadores, de allí han llegado a incluir la seguridad industrial e higiene ocupacional con la finalidad de plantear las medidas a tomar para realizar las labores bajo condiciones óptimas, con disminución del riesgo e incremento de la seguridad, incidiendo como consecuencia, en una mayor productividad, abatiendo incapacidades temporales o permanentes, accidentes 0 enfermedades ocupacionales (Hernández, 2005).

Las opiniones anteriores están orientadas a mostrar la necesidad de las empresas de tener un plan dirigido a mantener óptimas condiciones laborales que garanticen la salud de los trabajadores, de este modo, los autores coinciden en el sentido de que de acuerdo con el lugar donde esté establecida la organización, deben apoyar la normativa a las leyes existentes para ello.

En este recorrido de ideas, la investigadora considera la seguridad industrial e higiene ocupacional como un sistema que abarca los procedimientos para identificar, evaluar y controlar los agentes nocivos y factores de riesgo, presentes en el medio ambiente laboral, los cuales deberán incorporarse en las gerencias de servicio lacustre de la industria petrolera división Occidente, a través de técnicas permanentes, sistemáticas, de mejora continua, bajo la aplicación de estrategias, en pro de la salud física, mental y emocional de los trabajadores en el ejercicio de su actividad laboral.

\section{Planificación en la gestión de seguridad industrial e higiene ocupacional}

En el proceso de planificación los gerentes en una organización, analizan y procesan información del entorno interno y externo, evaluando las diferentes situaciones vinculadas a la ejecutoría organizacional para prever y decidir sobre la direccionalidad futura. Por lo tanto, se puede afirmar que la planificación se convierte en un instrumento de gestión, de negociación y de control organizacional.

Según Terry (2007), la planificación consiste en seleccionar información y hacer suposiciones respecto al futuro para formular las actividades necesarias para realizar los objetivos organizacionales. Por otro lado, Chiavenato (2009) define a la planificación como una técnica para minimizar la incertidumbre y dar más consistencia al desempeño de la empresa.

En este orden de ideas, Swing (2009) considera que la planificación es en gran medida la tarea de hacer que sucedan cosas que de otro modo no sucederían. Asimismo, Castellano (2009) expresa que la planificación es un proceso interactivo para la toma de decisiones empleando los recursos disponibles, minimizando costos y maximizando beneficios, para lograr un fin determinado. Donde, cada organización cuenta con recursos financieros, humanos, culturales.

En estos términos, la planificación se preocupa por el qué hacer y no por cómo hacer; y además propende a la implementación de todos los medios necesarios para ponerla en práctica. Como estos medios incluyen toda la organización, es necesario atribuir responsabilidades a todos los niveles o subsistemas de la empresa: nivel 
institucional, nivel intermedio y nivel operacional.

A este respecto, Koontz (2003) manifiesta que la planificación se refiere a las acciones llevadas a cabo para realizar planes y proyectos de diferente índole. En este proceso se pueden cambiar muchas cosas con el tiempo. Por ello, una planificación debe ser exacta en lo que se quiere lograr, sigue un conjunto de pasos que se establecen inicialmente, y quienes realizan la planificación hacen uso de las diferentes expresiones y herramientas para reforzar las políticas y procedimientos de la planeación.

Ahora bien, en el contexto de la seguridad industrial e higiene ocupacional, la Norma COVENIN 4001 (2000) refiere que la planificación consiste en establecer una manera debidamente organizada para conseguir un objetivo determinado, estableciendo plazos y medios, con la amplitud que se requiere. De acuerdo con esta definición, el fin de la planificación de la prevención dentro del Sistema de Gestión, Seguridad Industrial e Higiene Ocupacional (SGSHO) es implantar las acciones necesarias para un eficiente control de los riesgos y esta debe establecer: (a)Cómo y cuándo hacerla y quién debe hacerla, a partir de los resultados de la revisión inicial de la acción preventiva y de la evaluación inicial de riesgos; (b) Objetivos y metas a conseguir, tanto en el conjunto del sistema como para cada uno de los niveles operativos de la estructura de la empresa que intervienen en la gestión del sistema; (c) Asignación de prioridades y plazos para los objetivos y metas establecidos; (d) Asignación de recursos, para lo que deberá tenerse en cuenta las responsabilidades definidas y la coordinación necesaria con otros sistemas de gestión propios de la empresa (producción, mantenimiento, calidad, ambiente); (e) Seguimiento periódico de la consecución de objetivos, mediante los canales de información adecuados y los indicadores representativos.
En función de lo planteado y a juicio de la investigadora, el proceso de planificación en la gestión de seguridad industrial e higiene ocupacional implica cumplir con todos los lineamientos y requisitos establecidos; mantener la integridad del sistema de seguridad cuando se aplican cambios en él. Durante el ciclo de planificación, se debe establecer un plan de acción para la implantación, el mejoramiento de la efectividad de cada uno de los elementos considerando la complejidad y los riesgos inherentes a sus actividades, productos y / o servicios, por lo cual se requiere conocer sus políticas, objetivos, además de las leyes y normas.

\section{Política}

Para la Norma Venezolana COVENIN 4004:2000 (COVENIN, 2000), la política representa el compromiso de la organización asumido por su más alto nivel ejecutivo, frente al colectivo de sus trabajadores, la sociedad, relativo a las directrices orientadas a la conservación, el desarrollo de los recursos físicos y humanos, así como a la reducción de los daños a la salud y a los bienes. Al respecto, Hernández (2005) señala lo que a continuación se cita:

Dentro de las políticas de seguridad está la implantación y el acatamiento de las normas de seguridad, las que tendrán prioridad sobre todas las normas de trabajo y producción. Éstas deben emerger directamente de la más alta jerarquía, darse a conocer a todos los funcionarios, técnicos y trabajadores de planta, así como reciente ingreso (Hernández, 2005, p. 42).

Para Muguerza (2010), las políticas en materia de seguridad industrial, son la consecuencia de la racionalidad, la filosofía y la cultura organizacional. Son reglas que se establecen para dirigir funciones 
y asegurar que estas se desenvuelvan de acuerdo con los objetivos deseados. Constituyen una orientación administrativa para impedir que los empleados desempeñen funciones que no desean o pongan en peligro el éxito de funciones específicas.

De acuerdo con Zúñiga (2004), como punto de partida de un sistema de gestión de seguridad, las directivas de toda compañía se deben pronunciar formalmente, a través de una política reflejando su interés por un trabajo realizado en forma segura y su compromiso hacia la salud ocupacional, posteriormente se definirán responsabilidades de todos los niveles de la organización en la implementación del programa y cumplimiento de todos los normativos que para esto haya lugar.

En el mismo orden de ideas, el Instituto Nacional de los Espacios Acuáticos (INEA) plantea lo que a continuación se expone:

La alta dirección debe definir la política que especifique claramente los objetivos generales del sistema de seguridad integral asegurándose que la misma sea adecuada al propósito de la organización, que incluye un compromiso de cumplir con los requisitos y de mejorar continuamente la eficacia del sistema. A su vez, proporciona un marco de referencia para establecer y revisar los objetivos de ésta, que es comunicada y entendida dentro de la organización y que es revisada para su continua adecuación (INEA, 2009, p. 20).

Desde estas perspectivas, a criterio de la investigadora, en la política de la gestión de seguridad industrial e higiene ocupacional debe haber un compromiso de los niveles gerenciales, pues son sus directrices las que permiten hacer cumplir los objetivos del sistema de manera cuantificable y coherente.

En el contexto de las gerencias de servicio lacustre de la industria petrolera división
Occidente., la política debe abordar por igual las preocupaciones de la dirección y de los trabajadores. Al mismo tiempo, de permitir una adecuada actividad y el reconocimiento de la seguridad e higiene ocupacional como parte integral de la gestión de la organización.

\section{Objetivo}

Según Chiavenato (2009), el objetivo es un resultado deseado que se pretende alcanzar dentro de un período determinado. En el mismo orden de ideas, Robbins y Coulter (2005), definen los objetivos como los resultados deseados para individuos, grupos y organizaciones enteras.

Dentro de la norma OHSAS 18001:2007 (OHSAS Project Group, 2007) se entiende por objetivo las metas que una organización se establece a fin de cumplirlas. Debe ser cuantificable cuando sea factible. De acuerdo con la Norma Venezolana COVENIN 4001 (2000), es un conjunto de fines que la organización se propone alcanzar y deben ser cuantificados y fechados.

Para Hernández et al. (2003), en el contexto de la seguridad industrial e higiene ocupacional su objetivo es prevenir los accidentes laborales, los cuales se producen como consecuencia de las actividades de producción, por lo tanto, una producción que no contempla las medidas de seguridad e higiene no es una buena producción. Una buena producción debe satisfacer las condiciones necesarias de los tres elementos indispensables, seguridad, productividad y calidad de los productos. Por tanto, contribuye a la reducción de sus socios y clientes.

Por su parte, Chiavenato (2007) acota que la higiene laboral tiene un carácter principalmente preventivo, pues su objetivo principal es la salud y la comodidad del empleado, cuando evita que este se enferme y se ausente provisional o definitivamente del trabajo. Dicho esto, se conceptualiza el término objetivo como el resultado deseado por la dirección de la empresa 
que se propone a alcanzar en una línea de tiempo definida a fin de aminorar los accidentes laborales.

\section{Leyes y normas}

Para Azcuénaga (2010), las leyes y normas se refieren a la legislación vigente que está obligada a cumplir la organización. Adicionalmente, la norma internacional OHSAS 18001:2007 (OHSAS Project Group, 2007) establece que las leyes y normas son requisitos legales aplicables, tomados en cuenta en el establecimiento, implementación y mantenimiento del sistema de gestión de seguridad y salud ocupacional.

En otra perspectiva, según Asfahl y Rieske (2010) se refieren a las leyes y normativas como un conjunto de normas obligatorias de cumplimiento y cuya violación incurre a penalizaciones. Ante lo antes descrito, se definen las leyes y normativas como los requisitos legales que influencian sobre el sistema de gestión de seguridad y salud ocupacional.

A efectos de la presente investigación son consideradas las siguientes normativas venezolanas: Ley Orgánica de Prevención, Condiciones y Medio Ambiente de Trabajo (LOPCYMAT, 2005), Reglamento Parcial de la LOPCYMAT (2007), Reglamento de las Condiciones de Higiene y Seguridad en el Trabajo, Norma Técnica Programas de Seguridad y Salud en el Trabajo (NT-01-2008) del INPSASEL y la Norma Internacional OHSAS 18001:2007.

Ley orgánica del trabajo, los trabajadores y las trabajadoras (2012), publicada en Gaceta Extraordinaria No. 6.076 en el año 2012 en su artículo 43 estipula: "Todo patrono [...] es responsable por los accidentes laborales ocurridos y enfermedades ocupacionales acontecidas a los trabajadores, trabajadoras, aprendices, pasantes, becarios y becarias en la entidad de trabajo, o con motivo de causas relacionadas con el trabajo".

Ley orgánica de prevención, condiciones y medio ambiente de trabajo (LOPCYMAT, 2005): La Ley Orgánica de Prevención Condiciones y Medio Ambiente de Trabajo es una ley publicada en la Gaceta Oficial de la República Bolivariana de Venezuela número 38.236, de fecha 26 de julio de 2005; cuyo objeto se encuentra contemplado en su artículo número 1 , el cual establece que las instituciones deben establecer normas y lineamientos que permitan garantizar a los trabajadores y trabajadoras, condiciones de seguridad, salud y bienestar en un ambiente de trabajo adecuado y propicio para el ejercicio pleno de sus facultades físicas y mentales.

Reglamento Parcial de la LOPCYMAT: Publicada en la Gaceta Oficial de la República Bolivariana de Venezuela número 38.596, de fecha 3 de enero de 2007, el Reglamento Parcial de la LOCYMAT tiene por objeto desarrollar las normas de la Ley Orgánica de Prevención, Condiciones y Medio Ambiente de Trabajo (LOPCYMAT, 2005).

Norma técnica programa de seguridad y salud en el trabajo (NT-01-2008): Esta norma técnica desarrollada por el Instituto Nacional de Prevención, Salud y Seguridad Laborales publicada el 01 de diciembre de 2008, tiene por objeto establecer los criterios, pautas y procedimientos fundamentales para el diseño, elaboración, implementación, seguimiento y evaluación de un Programa de Seguridad y Salud en el Trabajo, con el fin de prevenir accidentes de trabajo y enfermedades ocupacionales en cada empresa.

Norma Internacional OHSAS 18001:2007 (OHSAS Project Group, 2007): Esta serie de normas OHSAS especifica los requisitos para un sistema de gestión de seguridad y salud ocupacional que permita a una organización controlar sus riesgos y mejorar su desempeño SySO. Estas habilitan a una empresa a formular una política y objetivos específicos asociados al tema, considerando los requisitos legales e información sobre los riesgos inherentes a su actividad.

En este recorrido de ideas, para la investigadora, las leyes y normas están referidas a un conjunto de procedimientos y estrategias legales destinados a preservar la integridad física de los trabajadores en el ejercicio de su actividad laboral. 


\section{MÉTODO}

La metodología fue de tipo descriptiva, bajo un diseño de campo, no experimental, y transeccional. La población estuvo conformada por las gerencias de Servicio Lacustre de la división occidente, específicamente en las Costas Occidental (Muelles Alí Primera, San Francisco, Sucre y Libertador) y Oriental del Lago (Muelles la Salina, Tía Juana, Simón Bolívar, Luisa Cáceres de Arismendi, Soberanía, Patria Grande, Rafael Urdaneta, Ciudad Ojeda, Lagunillas Norte, Lagunillas Sur y Bachaquero I), cuyos sujetos informantes fueron quince (15) gerentes y quince (15) supervisores de SIAHO.
Para la recolección de datos se utilizó la encuesta mediante la aplicación de un cuestionario, el mismo quedó conformado por 9 ítems con escala dicotómica. La validez se realizó a través del juicio de expertos, y para calcular su confiabilidad se empleó el método de Küder Richardson, con el que se obtuvo una confiabilidad de 0,85 para el instrumento al incluir todos los ítems.

El análisis de los datos se realizó mediante la estadística descriptiva, a través del estudio de las frecuencias relativas por medio de la aplicación del baremo de interpretación correspondiente, mostrado en la Tabla 1.

Tabla 1. Baremo de interpretación.

\begin{tabular}{ccc}
\hline Alternativa de respuesta & Intervalo & Categoría \\
\hline Si & $75 \% \leq \mathrm{X}<100 \%$ & Alta presencia \\
$\mathrm{Si}$ & $50 \% \leq \mathrm{X}<75 \%$ & Moderada presencia \\
$\mathrm{Si}$ & $25 \% \leq \mathrm{X}<50 \%$ & Baja presencia \\
$\mathrm{Si}$ & $0 \% \leq \mathrm{X}<25 \%$ & Muy Baja presencia \\
\hline
\end{tabular}

RESULTADOS

En la Tabla 2 se muestran los resultados para el indicador políticas, de la dimensión proceso de planificación, correspondientes a los ítems 1,2 y 3 del instrumento. De acuerdo al baremo establecido, en las gerencias de servicio lacustre de la industria petrolera venezolana división
Occidente, se tiene alta presencia de las políticas, situación que se devela al ubicarse en promedio el $80 \%$ de respuestas en la opción si, denotando alta presencia de un conjunto de reglas que se establecen para dirigir funciones y asegurar que estas se desenvuelvan de acuerdo con los objetivos deseados.

Tabla 2. Indicador: Políticas.

\section{Ítems}

1 ¿La empresa implementa normas de seguridad industrial e higiene ocupacional?

2 ¿Se establecen reglas para asegurar que estas se desenvuelvan de acuerdo con los objetivos deseados?

3 ¿La gerencia cuenta con una política dirigida a la seguridad industrial e higiene ocupacional? 


\section{Ítems}

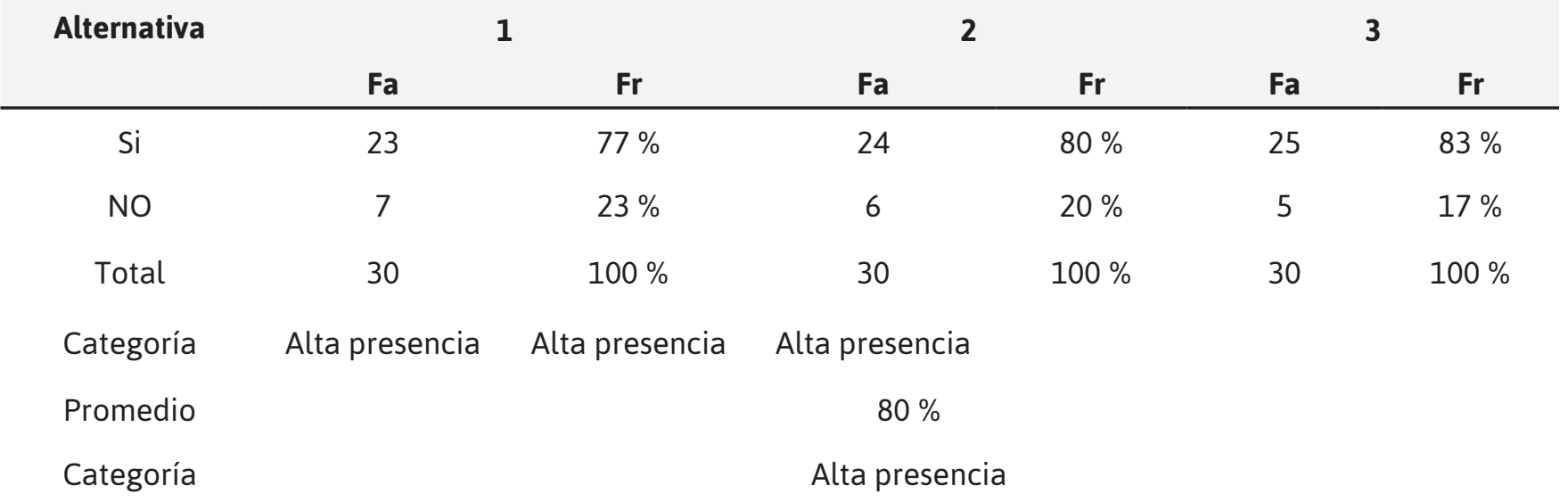

Estos resultados reflejan que en las gerencias de servicio lacustre, se puede apreciar que en el indicador políticas los encuestados atribuyeron alta presencia, con un $77 \%$ en la opción si, al preguntarles, tal como se evidencia en la Tabla 2 , si se implementan normas de seguridad industrial e higiene ocupacional, se establecen reglas para asegurar que estas se desenvuelvan de acuerdo con los objetivos deseados ( $80 \%$ ) y se cuenta con una política dirigida a la seguridad industrial e higiene ocupacional (83\%).

Por lo que a juicio de la investigadora en las gerencias bajo estudio la política debe abordar por igual las preocupaciones de la dirección y de los trabajadores. Al mismo tiempo, permitir una adecuada actividad y el reconocimiento de la seguridad e higiene ocupacional como parte integral de la gestión de la organización.

Los resultados evidenciados validan los postulados teóricos del Instituto Nacional de los Espacios Acuáticos (INEA) (2009), que plantea que la alta dirección debe definir la política que especifique claramente los objetivos generales del sistema de seguridad integral asegurándose que la misma sea adecuada al propósito de la organización, que incluye un compromiso de cumplir con los requisitos y de mejorar continuamente la eficacia del sistema.

Asimismo, alcanzan alta coincidencia con lo expuesto por la investigadora al afirmar que la política de la gestión de seguridad industrial e higiene ocupacional debe contemplar el compromiso de los niveles gerenciales, pues son sus directrices las que permiten hacer cumplir los objetivos del sistema de manera cuantificable y coherente.

Los resultados mostrados en la Tabla 3 correspondenal indicador objetivo, de la dimensión proceso de planificación. Se aprecia que, en promedio, el $72 \%$ de los encuestados consideran que las actividades indicadas en los ítems se realizan con moderada presencia. A detalle se muestra moderada presencia con respecto a que los objetivos son establecidos de acuerdo a los lineamientos de la política de seguridad industrial e higiene ocupacional de la empresa (63\%), y que los objetivos de la empresa orientan la toma de decisiones (73\%). Mientras que denotaron con alta presencia ( $80 \%$ de los encuestados) el hecho de que se definen los objetivos estratégicos para la prevención de accidentes laborales.

Estos resultados son altamente favorables para la gestión por cuanto queda en evidencia que en las gerencias de servicio lacustre de la industria petrolera división Occidente, moderadamente se aplican un conjunto de fines que la organización se propone a alcanzar y que deben ser cuantificados

ISSN: 2788 - 6557| ISNN-L: 2788 - 6557
Volumen 3 | No. 2 | Julio - diciembre 2021
www.revistapanel.org


Tabla 3. Indicador: Objetivo.

\begin{tabular}{|c|c|c|c|c|c|c|}
\hline Ítems & & & & & & \\
\hline $\begin{array}{l}4 \text { ¿Los objetivo } \\
\text { ocupacional de }\end{array}$ & $\begin{array}{l}\text { stablecidos } \\
\text { presa? }\end{array}$ & erdo con los & ientos de lá & ca de se & id inc & e higiene \\
\hline 5 ¿Los objetivo & empresa ori & la toma de de & es? & & & \\
\hline 6 ¿Se definen l & tivos estraté & dara la preve & e accident & prales? & & \\
\hline & & & Ítems & & & \\
\hline Alternativa & & & & & & \\
\hline & $\mathrm{Fa}$ & $\mathbf{F r}$ & $\mathbf{F a}$ & Fr & $\mathbf{F a}$ & $\mathbf{F r}$ \\
\hline Si & 19 & $63 \%$ & 22 & $73 \%$ & 24 & $80 \%$ \\
\hline NO & 11 & $37 \%$ & 8 & $27 \%$ & 6 & $20 \%$ \\
\hline Total & 30 & $100 \%$ & 30 & $100 \%$ & 30 & $100 \%$ \\
\hline Categoría & $\begin{array}{l}\text { Moderada } \\
\text { presencia }\end{array}$ & $\begin{array}{l}\text { Moderada } \\
\text { presencia }\end{array}$ & $\begin{array}{c}\text { Alta } \\
\text { presencia }\end{array}$ & & & \\
\hline Promedio & & & $72 \%$ & & & \\
\hline Categoría & & & erada pres & & & \\
\hline
\end{tabular}

Dado los resultados mostrados, se evidencia parcial congruencia con lo planteado en la teoría de Hernández et al (2003), pues en el contexto de la seguridad industrial e higiene ocupacional, el objetivo consiste en prevenir los accidentes laborales, los cuales se producen como consecuencia de las actividades de producción. Por su parte Chiavenato (2007), acota que la higiene laboral tiene un carácter principalmente preventivo, pues su objetivo principal es la salud y la comodidad del empleado, cuando evita que este se enferme y se ausente provisional o definitivamente del trabajo.

También validan, de manera moderada, lo considerado por la investigadora cuando plantea que los objetivos son establecidos como el resultado deseado por la dirección de la empresa que se propone a alcanzar en una línea de tiempo definida a fin de aminorar los accidentes laborales. Seguidamente, en la Tabla 4, se muestran los valores obtenidos de las respuestas correspondientes a los ítems relacionados al indicador leyes y normas. Se observa que, en promedio, el $54 \%$ de los encuestados consideran que las actividades involucradas en los ítems se realizan con moderada presencia. Específicamente se observó, según opinión de los encuestados, baja presencia de condiciones dignas para el desarrollo del trabajo (43\%), y de haber sido sancionada por el incumplimiento del reglamento de las condiciones de higiene y seguridad en el trabajo (43\%); no obstante, con alta presencia las gerencias se hacen responsable por los accidentes laborales y enfermedades ocupacionales ocurridos a los trabajadores (77\%). 
Tabla 4. Indicador: Leyes y normas.

Ítems

7 ¿Cuenta la empresa con condiciones dignas para el desarrollo del trabajo?

8 ¿La empresa se hace responsable por los accidentes laborales y enfermedades ocupacionales ocurridos a los trabajadores?

9 ¿La empresa ha sido sancionada por el incumplimiento del reglamento de las condiciones de higiene y seguridad en el trabajo?

\begin{tabular}{|c|c|c|c|c|c|c|}
\hline \multirow{3}{*}{ Alternativa } & \multicolumn{6}{|c|}{ Ítems } \\
\hline & \multicolumn{2}{|c|}{7} & \multicolumn{2}{|l|}{8} & \multicolumn{2}{|c|}{9} \\
\hline & $\mathrm{Fa}$ & Fr & $\mathrm{Fa}$ & $\mathbf{F r}$ & $\mathrm{Fa}$ & $\mathrm{Fr}$ \\
\hline $\mathrm{Si}$ & 13 & $43 \%$ & 23 & $77 \%$ & 13 & $43 \%$ \\
\hline NO & 17 & $57 \%$ & 7 & $23 \%$ & 17 & $57 \%$ \\
\hline Total & 30 & $100 \%$ & 30 & $100 \%$ & 30 & $100 \%$ \\
\hline Categoría & Baja presencia & Alta presencia & Baja presencia & & & \\
\hline Promedio & & & $54 \%$ & & & \\
\hline Categoría & & & Moderada presencia & & & \\
\hline
\end{tabular}

Estos resultados evidencian que las gerencias de servicio lacustre analizadas deben establecer los criterios, pautas y procedimientos fundamentales para el diseño, elaboración, implementación, seguimiento y evaluación de un programa de seguridad y salud en el trabajo, con el fin de prevenir accidentes y enfermedades ocupacionales.

Los resultados no alcanzan a validar en su totalidad lo expuesto por La Ley Orgánica de Prevención Condiciones y Medio Ambiente de Trabajo publicada en la Gaceta Oficial de la República Bolivariana de Venezuela número 38.236 , de fecha 26 de julio de 2005 , donde se expresa que las instituciones deben establecer normas y lineamientos que permitan garantizar a los trabajadores y trabajadoras, condiciones de seguridad, salud y bienestar en un ambiente de trabajo adecuado y propicio para el ejercicio pleno de sus facultades físicas y mentales.

Tampoco son totalmente congruentes con la posición de la investigadora, para quien las leyes y normas están referidas a un conjunto de procedimientos y estrategias legales destinados a preservar la integridad física de los trabajadores en el ejercicio de su actividad laboral.

Finalmente, en la Tabla 5 sepresenta el resumen para la dimensión proceso de planificación, reflejando, de acuerdo con los encuestados, una concentración de respuestas afirmativas del $69 \%$, indicando que se da moderada presencia del proceso de planificación en la gestión de seguridad industrial e higiene ocupacional en las gerencias de servicio lacustre de la industria petrolera venezolana división Occidente.

Tabla 5. Dimensión: Proceso de planificación.

\begin{tabular}{ccccc}
\hline Indicador & Promedio & Categoría & Promedio & Categoría \\
\hline Política & $80 \%$ & Alta presencia & & \\
Objetivo & $72 \%$ & Moderada presencia & $\mathbf{6 9 \%}$ & $\begin{array}{c}\text { Moderada } \\
\text { presencia }\end{array}$ \\
Leyes y normas & $54 \%$ & Moderada presencia & & \\
\hline
\end{tabular}


Al ver los resultados se describe con alta presencia la política, moderada presencia para el objetivo, así como las leyes y normas. A juicio de la investigadora estos aspectos deben ser considerados como oportunidades de mejora por cuanto el fin de la planificación de la prevención dentro de la seguridad industrial e higiene ocupacional es implantar las acciones necesarias para un eficiente control de los riesgos.

Lo develado valida lo expuesto por la Norma COVENIN 4001 (2000), la cual refiere que la planificación consiste en establecer una manera debidamente organizada para conseguir un objetivo determinado, estableciendo plazos y medios, con la amplitud que se requiere.

Al igual que es coincidente con lo expresado por la investigadora, cuando afirma que el proceso de planificación en la gestión de seguridad industrial e higiene ocupacional implica cumplir con todos los lineamientos y requisitos establecidos; mantener la integridad del sistema de seguridad cuando se implementan cambios en él, por lo cual se requiere conocer sus políticas, objetivos, además de las leyes y normas.

\section{CONCLUSIONES}

En relación al objetivo, donde se describió el proceso de planificación en la gestión de seguridad industrial e higiene ocupacional en las gerencias de servicio lacustre de la industria petrolera venezolana, se concluye que estas gerencias poseen moderada presencia de este proceso en la gestión de seguridad industrial e higiene ocupacional que realizan. Debe mencionarse que, dada esta categoría de moderada presencia, donde se detectó la existencia de ciertos aspectos establecidos como determinantes para medir esta dimensión, los mismos deben ser reforzados a fin de ofrecer oportunidades de mejora.

Específicamente en lo que concierne a política, se concluye que en las gerencias bajo estudio se debe abordar por igual las preocupaciones de la dirección y de los trabajadores. Al mismo tiempo, permitir una adecuada actividad y el reconocimiento de la seguridad e higiene ocupacional como parte integral de la gestión de la organización. Respecto al indicador objetivo, se obtuvo que no son tan favorables para la gestión por cuanto queda en evidencia que en las gerencias de servicio lacustre de la industria petrolera venezolana división Occidente, moderadamente, se aplican un conjunto de fines que la organización se propone a alcanzar y que deben ser cuantificados.

Con respecto al indicador leyes y normas, los resultados dan evidencia que las gerencias de servicio lacustre analizadas deben establecer los criterios, pautas y procedimientos fundamentales para el diseño, elaboración, implementación, seguimiento y evaluación de un programa de seguridad y salud en el trabajo, con el fin de prevenir accidentes y enfermedades ocupacionales.

\section{REFERENCIAS}

Aguilar, E. (2011). Desarrollo de un modelo de gestión de seguridad e higiene industrial. Primera edición. Editorial EAE. España

Asfahl, R. y Rieske, D. (2010). Seguridad industrial y la administración de la salud. Editorial: Pearson. México

Azcuénaga, L. (2010). Guía para la Implantación de un Sistema de Prevención de Riesgos Laborales, FC Editorial, Cuarta edición. Madrid

Burgos, A. (2008). Seguridad y sentido común, higiene ocupacional. Disponible en: http:// www.mailxmail.com/autor-abraham-burgos. Consultado el: 10/01/2020

Camisón, C., Cruz, S. y González, T. (2007) Gestión de la Calidad: Conceptos, enfoques, modelos y sistemas. Ediciones Pearson Educación, S.A Madrid-España

Castellano, B. (2009). Planificación. CENDES. Cuarta Edición. Caracas -Venezuela

Chiavenato, I (2001). Administración. Proceso Administrativo. Editorial McGraw-Hill Interamericana Editores S.A. Bogotá 
Chiavenato, I. (2007). Administración de Recursos Humanos. El Capital Humano de las Organizaciones. Mc Graw-Hill. Venezuela

Chiavenato, I. (2009) Introducción a la Teoría Administrativa. Editorial Mc Graw Hill. México

Chinchilla, R. (2011) Salud y seguridad en el trabajo. 5ta. Edición. EUNED. Costa Rica

Constitución de la República Bolivariana de Venezuela (1999). Gaceta Oficial número 36.860 de la República Bolivariana de Venezuela. Disponible en: http://www.mp.gob. ve/LEYES/constitucion/constitucionl.html Consultado el: 09/12/2019

Duque, B. (2010). Agenda ambiental para el sector minero. Fundación AVINA. Colombia. Bogotá

Hernández, A. (2005) Seguridad e Higiene Industrial. Editorial Limusa. México

Hernández, A.; Malfavón, N.; y Fernández, G. (2003). Seguridad e higiene industrial. Editorial Limusa. Mexico

Hernández, A; Zúñiga, N; Malfavon, G. (2005). Seguridad e Higiene Industrial. 6ta Edición. Editorial Limusa. México

Instituto Nacional de los Espacios Acuáticos (INEA) (2009). Sistema de gestión de seguridad integral para operaciones portuarias (SISEINOP). Disponible en: http://mismisiones.blogspot. com/2009/03/seguridad-integral-paraoperaciones.html. Consultado el: 24/11/2019

Koontz, H. (2003). Administración: una perspectiva global. Duodécima edición. Editorial McGraw Hill Interamericana. México

Ley Orgánica de Prevención, Condiciones y Medio Ambiente de Trabajo, (LOPCYMAT, 2005). Disponible en: http://www.inpsasel. gob.ve/moo_doc/lopcymat.pdf Consultado: 09/10/2019

Ley orgánica del trabajo, los trabajadores y las trabajadoras (2012). Disponible en: http:// www.lottt.gob.ve/ Consultado el: 09/12/2019

Moreno, M. (2010) Manual para la formación en prevención de riesgos laborales: programa formativo para el desempeño de las funciones del nivel básico. 6ta. Edición. LEX Nova. España
Muguerza, J. (2010). Gerencia de seguridad industrial. Disponible en: http://www. emagister.com/curso-gerencia-seguridadindustrial/objetivos-gerencia-seguridadindustrial Consultado: 08/12/2019

Norma técnica programa de seguridad y salud en el trabajo (NT-01-2008). Disponible en: http://www.inpsasel.gob.ve/moo_doc/NOR_ TEC_PRO_SEG_SAL_TRA.pdf Consultado el: 09/12/2019

Norma Venezolana COVENIN 4001 (2000). Sistema de Gestión de Seguridad e Higiene Ocupacional. Requisitos. Disponible en; http:// www.cpzulia.org/ARCHIVOS_SSA/4001_2000_ SGSHO.pdf Consultado el: 09/12/2019

Norma Venezolana COVENIN 2260 (2004). Sistema de Gestión de Seguridad e Higiene Ocupacional. Requisitos: Disponible en: http://www.sencamer.gob.ve/sencamer/ normas/2260-04.pdf. Consultado: 08/12/2019

Norma Venezolana COVENIN 4004 (2000). Sistema de Gestión de Seguridad e Higiene Ocupacional. Requisitos: Disponible en: http:// www.cpzulia.org/ARCHIVOS_SSA/4004_2000_ Guia_SGSHO.pdf. Consultado: 08/12/2019

OHSAS Project Group (2007). Norma Internacional OHSAS 18001:2007 -Requisitos. Disponible en: $\quad$ https://manipulaciondealimentos.files. wordpress.com/2010/11/ohsas-18001-2007. pdf Consultado: 08/12/2019

PDVSA página oficial de internet (2019). Disponible en: http://www.pdvsa.com/index. php?tpl=interface.sp/design/readmenuprinc_ eps.tpl.html\&newsid_temas $=220$ Consultado el: 02/12/2019

Peralta U. (2010). Seguridad e higiene industrial. Editorial Mc Graw Hill. México

Reglamento Parcial de la LOPCYMAT (2007). Disponible en: http://www.inpsasel.gob.ve/ moo_doc/regl_par_lopcymat.pdf Consultado el: 09/12/2019

Rodríguez, J. (2010) Administración de pequeñas y medianas empresas. 6ta. Edición. Cengage Learning Editores. México 
Swing, D. (2009). Principios de la planificación. Editorial Mc Graw Hill. D.F., México

Terry, G. (2007). Principios Administrativos. Editorial El Ateneo. Buenos Aires. Argentina

Villegas, J. (2008). Administración de Personal. Editorial Mc Graw Hill. México
Zúñiga, L. (2004). Reducción de los accidentes del trabajo mediante el Cambio de la conducta hacia la seguridad. Revista Mapfre Seguridad España, 13(52), 31-38 